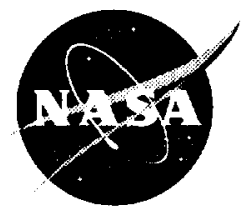

\title{
Qualitative Flow Visualization of a 110-N Hydrogen/Oxygen Laboratory Model Thruster
}

Wim A. de Groot

NYMA, Inc., Brook Park, Ohio

Thomas J. McGuire

Pennsylvania State University, University Park, Pennsylvania

Steven J. Schneider

Lewis Research Center, Cleveland, Ohio

Prepared for the 33rd Joint Propulsion Conference and Exhibit cosponsored by AIAA, ASME, SAE, and ASEE

Seattle, Washington, July 6-9, 1997

National Aeronautics and

Space Administration

Lewis Research Center 
Available from

NASA Center for Aerospace Information 800 Elkridge Landing Road

Lynthicum, MD 21090-2934

Price Code: A03
National Technical Information Service 5287 Port Royal Road Springfield, VA 22100 Price Code: A03 


\title{
Qualitative Flow Visualization of a 110 N Hydrogen/Oxygen Laboratory Model Thruster
}

\author{
Wim A. de Groot ${ }^{*}$ \\ NYMA, NASA LeRC \\ Brook Park, $\mathrm{OH}$ \\ Thomas J. McGuire ${ }^{\dagger}$ \\ Pennsylvania State University \\ University Park, PA \\ Steven J. Schneider ${ }^{\ddagger}$ \\ NASA LeRC \\ Cleveland, $\mathrm{OH}$
}

\begin{abstract}
The flow field inside a $110 \mathrm{~N}$ gaseous hydrogen/oxygen thruster was investigated using an optically accessible, two-dimensional laboratory test model installed in a high altitude chamber. The injector for this study produced an oxidizer-rich core flow, which was designed to fully mix and react inside a fuel-film sleeve insert before emerging into the main chamber section, where a substantial fuel film cooling layer was added to protect the chamber wall. Techniques used to investigate the flow consisted of spontaneous Raman spectra measurements, visible emission imaging, ultraviolet hydroxyl spectroscopy, and high speed schlieren imaging. Experimental results indicate that the oxygen rich core flow continued to react while emerging from the fuel-film sleeve, suggesting incomplete mixing of the hydrogen in the oxygen rich core flow. Experiments also showed that the fuel film cooling protective layer retained its integrity throughout the straight section of the combustion chamber. In the converging portion of the chamber, however, a turbulent reaction zone near the wall destroyed the integrity of the film layer, a result which implies that a lower contraction angle may improve the fuel film cooling in the converging section and extend the hardware lifetime.
\end{abstract}

\footnotetext{
* Sr. Research Engineer, Senior Member AIAA †Undergraduate Student, Student Member AIAA $\ddagger$ Aerospace Engineer, Senior Member AIAA
}

\section{Introduction}

The performance of small chemical thrusters, (less than $200 \mathrm{~N}$ thrust), generally deteriorates with decreasing thruster size and thrust level. The reasons for this trend are directly related to the small physical size of the thrusters, which result in combustion and flow behavior different from large rocket engines.

In order to optimize small rocket performance and life, all the combustion processes have to be completed before the high temperature product mixture is expelled through the nozzle. For this to occur, critical processes, such as propellant mixing (critical for gaseous injection), evaporation (critical for liquid injection), and reactions must be completed. Combustion needs to occur far enough from the injector to prevent overheating or melting of the injector face. A compromise between these two criteria often leads to injector designs which exhibit incomplete combustion.

Because of the large surface-to-volume ratio of small combustion chambers, a significant fraction of the heat of combustion is transferred to the walls. In order to keep wall material temperatures below thermal limits, as well as 
protect it from oxidation, fuel film cooling is usually employed in small rockets. In some instances, a substantial portion of the fuel is used for film cooling. Although some of the fuel film will react with the oxidizer-rich core flow, most of it will leave the combustor unreacted, contributing to performance losses.

Because of the small throat and nozzle dimensions, the nozzle boundary layer is relatively thick compared to large rockets. The large boundary layer results in relatively large viscous losses, degrading nozzle performance. As a result of this disproportionally large viscous domain, the nozzle optimization for small rockets is not as well-defined as for large rockets. '

Numerical tools are often used for a quick examination of performance and life trends as a function of design changes. The phenomena described above make it difficult to predict the performance and thermal behavior of small rockets, and thus call into question numerical results. Richter and Price, ${ }^{2}$ for example, use a standard JANNAF methodology to predict the specific impulse $\left(\mathrm{I}_{\mathrm{sp}}\right)$ of a $220-\mathrm{N}$ gaseous hydrogen/ oxygen $\left(\mathrm{GH}_{2} / \mathrm{GO}_{2}\right)$ thruster operating at various mixture ratios. They show an overprediction of as much as $18.2 \%$ at an oxygen to fuel $(\mathrm{O} / \mathrm{F})$ ratio of 7 . Weiss ${ }^{3}$ uses the NavierStokes based axisymmetric RPLUS code, including a $k-\varepsilon$ turbulence model, to predict performance parameters of two $110 \mathrm{~N}$ thrusters. Predicted values for one thruster, operating at an overall $\mathrm{O} / \mathrm{F}$ ratio of 6.8 with a fuel-film cooling (FFC) percentage of $15 \%$, are higher by $23 \%$ in thrust and $18 \%$ in $\mathrm{I}_{\mathrm{sp}}$ than measured values.

This study described here was undertaken to provide a better understanding of the flow field behavior in small rockets and to provide a data base to anchor numerical codes. The objective was to visualize the reacting flow field and to qualitatively establish flow patterns, reaction zones, and if possible, species concentrations. Measurements were made with a $110-\mathrm{N}$ $\mathrm{GH}_{2} / \mathrm{GO}_{2}$ injector operating on an optically accessible, two-dimensional combustion chamber. In addition to previously reported spontaneous Raman scattering data, flow field emission and Schlieren visualization were performed. Flow visualization images were processed with frame-grabber software into semiquantitative data that could be used to compare with the Raman scattering data.

\section{Hardware}

A two-dimensional laboratory model thruster, shown schematically in Fig. 1, was used in all experiments performed in this study. The $\mathrm{GH}_{2} / \mathrm{GO}_{2}$ injector used was originally developed for a space station propulsion technology program. ${ }^{4}$ The combination of injector and fuelfilm sleeve insert was essentially a spark torch igniter, where an oxidizer-rich flow created inside the sleeve, was used to ignite the main propellants in a larger chamber. In this case, the torch igniter served as the oxygen-rich "preburner" of a 110-N thruster. Oxygen was radially injected in the plenum behind the spark plug tip (see Fig. 1a). The oxygen flowed past the blunt spark plug tip, where it was energized. Hydrogen was radially injected just downstream of the spark plug tip to produce an oxygen-rich core flow. The core flow was ducted into the chamber sleeve insert, which had a coannular flow of the remaining hydrogen for film cooling. For the two-dimensional thruster, a square sleeve insert was made with slots on the top and bottom for fuel film cooling.

The square rocket chamber was fabricated from OFHC copper and was water-cooled on the top and bottom, as shown in Fig. 1b. Fused silica windows were installed in the sides of the chamber to provide optical access from the exit of the sleeve insert to the nozzle exit. The windows were protected from the combustion process by a gaseous nitrogen shroud. The cooling nitrogen was parallel with the windows to avoid nitrogen penetration into the core until downstream of the sleeve exit. The combustion chamber contraction ratio, throat diameter, and nozzle expansion ratio were dimensionally similar to the axi-symmetric thruster that the injector was originally designed for. Figure 2 shows a photograph of the square chamber sleeve insert and the two-dimensional chamber.

All of the thruster testing was conducted in a high-altitude facility capable of an ambient pressure of $1 \mathrm{kPa}$. Details about the facility can be found in Reference 5. Testing was performed at a mixture ratio 6.0 and a chamber pressure of $286 \mathrm{kPa}$. The chamber pressure was the result of throat selection and represented only $60 \%$ of the real test article chamber pressure. The hydrogen flow split provided 55 percent fuel film cooling, which results in a core mixture ratio of 13.3. Because the facility diffuser did not adequately 
capture the plume from the square nozzle, hydrogen (from film cooling) accumulated in the altitude chamber. As a result, test durations were limited to 15 seconds.

\section{Diagnostics}

Spontaneous Raman Scattering

Spontaneous Raman scattering (SRS) was chosen over methods that would provide stronger signal to noise ratios (SNR), such as Coherent AntiStokes Raman Scattering (CARS), because of restrictions imposed by the geometry and the environment inside the high altitude simulation facility. Furthermore, SRS was amenable to implementation in an optical fiber configuration and thus permitted access to the thruster installed inside the high altitude simulation facility while satisfying laboratory safety rules.

The Raman measurement uses the spectral content of light scattered when a mixture of molecules is exposed to incident, monochromatic light, such as laser light. The molecules exchange energy with the incident field during the scattering process. The fraction of the light scattered with the same frequency as the incident light is referred to as Rayleigh scattering and is not species specific. A fraction of the light is scattered with a combination frequency of the incident frequency and internal molecular rotational-vibrational frequencies. Depending on whether the molecule has lost energy or gained energy from the interaction with the scattered light quantum, these light fractions are called anti-Stokes and Stokes scattering, respectively. The SRS signal is species specific and is used to identify molecules, and measure their quantities and temperatures.

For gaseous $\mathrm{GH}_{2} / \mathrm{GO}_{2}$ thrusters, the species to be detected and analyzed are hydrogen, oxygen, and water. Other combustion intermediates and products will not appear long enough or in sufficient quantity to be detected via SRS. Spectral modeling of species focused on the above listed species as well as on nitrogen which, due to its presence in air, is an excellent calibration gas.

A spectral simulation code coupled with a numerical curve fitting routine was written for the diatomic species and water. The code included the effect of light polarization and geometrical configuration of the optics on the individually detected line strengths, which is more general than the conventionally used "Raman Scattering Cross Section", A generic approach to such calculations for diatomic molecules is given by James and Klemperer. ${ }^{6}$ An excellent derivation for hydrogen is given by Shirley. ${ }^{\text {Th }}$ Temperature and number densities can be extracted by performing a LevenburgMarquardt best-fit analysis of the measured spectra with computational spectra.

A detailed description of the SRS system is given in Reference 8. A flashlamp-pumped dye laser, using Rhodamine 590 dye, generated SRS. The laser beam was optimized to a pulse energy of about $1.2 \mathrm{~J} /$ pulse with a full width at half maximum (FWHM) bandwidth of $0.03 \mathrm{~nm}(0.8$ $\left.\mathrm{cm}^{-1}\right)$. The total pulse length was $2.2 \mu \mathrm{s}$.

The temporal decay of the laser pulse energy was measured at the laser exit and then accounted for. The laser pulse was coupled by means of a microscope objective into a $800-\mu \mathrm{m}$ optical fiber with metal jacket. The fiber guided the laser pulses through the blast wall that separated the clean room from the test facility and through the bulkhead of the high altitude simulation chamber. In the chamber, the pulses were collimated and then refocused. Collection optics were positioned at $90^{\circ}$ from the focusing optics for the collection of Raman scattered light, which is shown in Fig. 1a. This geometry reduced the chance of collecting stray laser light. The confluence of delivery and collection optics created a probe volume of about $0.7 \mathrm{~mm}^{3}$. Baffles were placed in the delivery and collection optics and a beam stop was positioned to capture the laser pulses (possible in the exit plane, not in the combustion chamber). This caused an additional reduction in interference of stray laser light.

The collection optics imaged the probe volume into a $800-\mu \mathrm{m}$ optical fiber. This fiber guided the scattered light back through the bulkhead and through the wall of the clean room. There the light was collimated and passed through a Raman notch filter, an optical filter with attenuation at the laser line of $10^{-6}$ and transmission at the Raman lines of more then $90 \%$. A lens was used to refocus the light into a 0.5 -meter spectrometer with a $1200-$ groove/mm grating. This gave a spectral dispersion of $1.6 \mathrm{~nm} / \mathrm{mm}$. 
A red-enhanced intensified linear array was used for spectral measurements; the array consisted of 700 active pixels of $25 \mu \mathrm{m}$. With a 1200 groove/mm grating, a spectral area of $28 \mathrm{~nm}$ was observed. The intensifier was gated in order to improve the SNR. The background-corrected spectra were stored in raw format and later corrected for laser pulse energy.

\section{Spectrally Filtered Emission}

An investigation of the emitted light was performed to provide information on the combustion flow field and the chemical reactions taking place. The use of spectral interference filters tailored to emission from known processes which occur in hydrogen-oxygen flames can provide information on specific mixing and reactions.

In addition, the extent to which the nitrogen window shroud participates in the core combustion was also examined by highlighting particular spectral regions with bandpass filters. Flames containing oxides of nitrogen show emission in the green and blue, a region where emission from hydrogen-oxygen combustion processes is very weak. Investigating the presence of green-yellow emission showed the extent of participation of nitrogen in the combustion processes.

A solid state camera with a $f / 11$ focusing lens was used to record the emission. Optical interference filters were placed in front of the camera, mounted outside the high altitude simulation chamber. The optical interference filters, centered at different wavelengths, had a bandpass of $20 \mathrm{~nm}$ FWHM. The filters used were based on the availability and spectral wavelength related to specific processes. The camera monitored the combustion process through the 25.4-mm thick fused silica viewport and through the 12.7-mm thick fused silica thruster widows. Because fused silica is transparent over the spectral range of 200 to $1100 \mathrm{~nm}$, the attenuation due to the windows was negligible. Images were obtained at a frequency of $30 \mathrm{~Hz}$ and stored on video tape.

\section{Ultraviolet Spectra}

The dominant spectral feature of hydrogen/oxygen flames is due to the $\mathrm{OH}$ radical. The strongest $\mathrm{OH}$ emission is in the ultraviolet, with a maximum intensity bandhead at $306.36 \mathrm{~nm}$. OH formation occurs at or near the flame front. A determination of the strength of the ultraviolet emission provides information on the location of the flamefront and the extend of the chemical reactions taking place.

High-resolution, spectrally-resolved, ultraviolet spectra of the hydrogen-oxygen flame were obtained. Because higher rotational levels become populated at higher temperatures, the intensity distribution over the well resolved rotational structure provides information on the rotational temperature. Furthermore, the overall intensity of the spectrum provides information on the number density of the hydroxyl radical.

Optics were assembled inside the high altitude simulation chamber to collect emission spectra from a $3 \mathrm{~mm}$ diameter cylindrical probe volume spanning the two-dimensional chamber, as shown in Fig. 1a. The probe volume was moved vertically in a plane $1.5 \mathrm{~mm}$ behind the sleeve exit plane. The collected light was coupled into a $20 \mathrm{~m}$ optical fiber which guided the light to a $1.26 \mathrm{~m}$ spectrometer installed inside a clean room. The 2400 groove $/ \mathrm{mm}$ blazed grating provided a spectral resolution of $0.325 \mathrm{~nm} / \mathrm{mm}$. A 700 active pixel diode array provided spectral images of $5.78 \mathrm{~nm}$.

Spectra were recorded from 300 to $337 \mathrm{~nm}$, by gluing 10 spectral sections together with some overlap to assure intensity and wavelength match. Each spectral was obtained with a array exposure of $14 \mathrm{sec}$, the duration of a test run.

\section{Schlieren Visualization}

Schlieren visualization was used to image density and temperature gradients in the combustion chamber. In the schlieren technique, collimated light is passed through a medium with a changing refractive index, such as a chemically reacting flow. Density and/or temperature changes in the medium cause these refractive index changes. A focusing lens is used to focus the light after it passes through the medium. Installing a knife edge at the focal point blocks the light that does not encounter a refractive index change, and thus is not deflected. Light that is deflected as the result of a refractive index change passes the knife edge and is imaged on a screen. This image highlights the refractive index changes, and thus shows the density and/or temperature gradients.

High speed recording of the images provides both a temporal and spatial resolved display of 
mixing and reactions. Hydrogen and oxygen have significantly different refractive indexes. Turbulent mixing of these two species causes refractive index gradients which can provide information on the mixing processes. Chemical reactions cause strong temperature gradients, which result in strong density gradients and thus refractive index gradients. These reaction regions are imaged with schlieren visualization.

A schlieren visualization system was assembled inside the test cell. All components were mounted outside the high altitude simulation chamber. The viewports were utilized for transmitting the light. A system schematic is shown in Fig. 3. The beam of a $5 \mathrm{mWatt}$ green HeNe laser, with wavelength $543.5 \mathrm{~nm}$, was expanded to a $76 \mathrm{~mm}$ diameter beam. A fully reflecting mirror deflected the beam into the altitude facility, where it passed through the optical quality quartz viewport and the two dimensional combustion chamber. The light exited the altitude chamber through the second optical quality quartz viewport. A refocusing lens imaged the light beam into a point image. A second lens, installed behind the focal point imaged the beam onto a ground glass screen, where the image was recorded with a 1000 frames/s intensified imager.

A quartz plate, supporting a $0.5-\mathrm{mm}$ diameter, flat black, circular mask was installed at the focal point. The purpose of the mask was to block all the light that passed through the two-dimensional chamber without being deflected. Most of the light that was deflected as the result of density gradients in the light path passed the mask and was imaged onto the screen. The circular mask was chosen over a knife edge because it displayed density gradients in both axial as well as radial directions, as compared to only radial directions for a horizontally placed knife edge. Changing the size of the dot mask changed the sensitivity of the system, allowing lesser or stronger density gradients to be detected.

The high speed images were recorded on a video recorder and analyzed using a image analyzer. Semi-quantitative radial density gradient profiles were obtained. The intensity distribution along a column of pixels was extracted from the image, and an intensity average of ten neighboring columns of pixels was obtained. With the image size recorded, this corresponded to a $1.4 \mathrm{~mm}$ axial resolution. In order to eliminate high frequency oscillations, twenty consecutive distributions with $1 \mathrm{msec}$ between images were averaged.

\section{Results}

Spontaneous Raman Scattering

Data from SRS are plotted in Figs. 4 and 5. The horizontal axis is the $\mathrm{z}$-coordinate and represents the distance from the center line in $\mathrm{mm}$. The distance is non-dimensionalized with respect to $h$, the half height of the square flow channel. The figures illustrate that a "cold", oxygen rich zone exists in the wake of the blunt spark plug. The temperature at this location was approximately $1770 \mathrm{~K}$. A temperature maximum was detected at a non-dimensional distance of 0.6 . The average temperature at that location was approximately $2800 \mathrm{~K}$. Due to the presence of the square channel wall, no measurements closer than $\mathrm{z} / \mathrm{h}=0.8$ could be made.

An oxygen number density maximum was detected the at the centerline. The oxygen number density decreased radially till a minimum was measured at $z / h=0.8$. Surprisingly, a local maximum was also found in the water density at the center line. A minimum water number density was found at $\mathrm{z} / \mathrm{h}=0.6$, after which the maximum was found at 0.8 . It should be noted that the water number density, expressed in mole fraction, looked significantly different than the water number density in molecules per volume, due to the high temperature at $\mathrm{z} / \mathrm{h}=0.6$. But because hydrogen and hydroxyl (OH) concentrations could not be measured, an exact mole fraction could not be extracted. These figures showed that a likely flame zone resided around $\mathrm{z} / \mathrm{h}=0.6$.

\section{Spectrally Filtered Emission}

The emission spectrum of the two-dimensional thruster was characterized by a bluish white color. At the pressures tested $(0.3 \mathrm{MPa})$, the emission was too intense to detect any variation across the chamber without spectral filters. Neutral density filters and interference filters were utilized in the visible part of the spectrum to reduce the intensity and to separate emission by wavelength. These spectral observations provided a tool to interpret combustion behavior.

Two emission images of the two-dimensional chamber are shown in Fig. $6 a$ and $6 \mathrm{~b}$. In these figures, the flow is imaged from the left to the 
right. The left boundary was the sleeve exit. These images were obtained with bandpass filters centered at 479 and $630 \mathrm{~nm}$, corresponding to spectral regions where oxygen and water, respectively, are dominant emitters.

Emission in the dark blue region (around 479 $\mathrm{nm}$ ) consisted of a continuous background level with local regions of slightly stronger emission. The core emission at this wavelength was significantly stronger than in other wavelength ranges investigated. This strong signal is most likely attributable to the Schumann-Runge band emission of oxygen, as described by Gaydon," which are strongest in the 300 to $400 \mathrm{~nm}$ range but extend a considerable range into the visible. The observation made by Gaydon that maximum emission lies fairly well towards the oxygen side of the hottest region explained the relatively strong emission in the core.

The slightly higher intensity near the wall of the converging section of the chamber in Fig. 6 a appeared to be caused by the formation of water via reaction:

$$
\mathrm{OH}+\mathrm{H}_{2} \rightarrow \mathrm{H}_{2} \mathrm{O}+\mathrm{hv}
$$

According to Gaydon, ${ }^{9}$ the emission produced in this reaction consists of a spectral continuum which extends from at least 220 through $600 \mathrm{~nm}$, but has a flat maximum at $450 \mathrm{~nm}$, thus contributing significantly in the $479 \mathrm{~nm}$ spectral region.

In the $630 \mathrm{~nm}$ spectral region, most emission appeared in the outer regions of the core and in the converging section near the wall. Differences in emission intensity across the chamber were much more significant than in the blue region. The likely cause was emission from the infrared vibration-rotation spectrum of $\mathrm{H}_{2} \mathrm{O}$, having a strong band head at $616.5 \mathrm{~nm}$ and degraded towards the longer wavelengths. According to Gaydon, ${ }^{9}$ experiments show that no especially strong emission occurs in the hydrogen/oxygen reaction zone, suggesting only thermal excitation of the $\mathrm{H}_{2} \mathrm{O}$. This observation provides a possible explanation why no sharply defined combustion region could be observed. Because the $616.5 \mathrm{~nm}$ band is associated with a higher vibrational excitation, a higher temperature region is more likely to produce this emission. A weak contribution could have come from the rotationvibration bands of $\mathrm{OH}$. A comparison near the sleeve, however, shows that the presence of $\mathrm{OH}$ does not coincide with the light intensity.

Fig. 6b shows a low emission core, extending from the sleeve exit to the throat. The measurements indicated either that the $\mathrm{H}_{2} \mathrm{O}$ concentration in this region was low or that the temperature was significantly below reaction zone temperature. A comparison with the Raman measurements of Fig. 3 suggests the former explanation. The low emission core zone extended throughout the length of the chamber, indicating little core mixing. This supported Raman measurements made on the centerline, inside the combustion chamber and throughout the throat, that showed low $(900-1200 \mathrm{~K})$ temperatures. ${ }^{10}$ A likely explanation of the lower measured core temperature near the throat as compared to near the sleeve exit was the diluting effect of the nitrogen window cooling. A slightly stronger emission in this zone was seen in the green-yellow spectral region. This was not as strong as anticipated, however, based on the quantity of nitrogen coolant.

The emission intensity in Fig. 6b shows a gradual increase from the dark core outward. This again supports the Raman data, which showed a lowest temperature in the core and a maximum temperature at a non-dimensional distance $\mathrm{z} / \mathrm{h}=0.6$ from the centerline. A clear maximum could not be observed. Near the sleeve exit, however, the intensity reached a maximum about two-thirds outward from the centerline, than decreased when approaching the top and bottom walls.

Near the sleeve exit, bordering the cooled walls, a region of low emission was observed. This showed the cold hydrogen-film injection. Moving downstream along the walls, the emission gradually increased, and reached a maximum about one third of the distance from the start of the converging section to the throat. This emission was more intense than anywhere else in the observable part of the chamber. A likely explanation is a source of high temperature water and/or hydroxyl. It is speculated that the hydrogen covering the walls does not have sufficient momentum to conquer the Coriolis forces and separates from the wall. Thus, a strong turbulent mixing region where the core and hydrogen mix and react is postulated. The consumption of the wall protective fuel-film and the high temperature of the reaction zone could 
present a problem by limiting the life of the thruster. Whether this phenomenon, observed in the two-dimensional chamber used for these experiments, actually occurs in an axisymmetric chamber is not clear.

The nitrogen shroud protecting the windows did not contribute significantly to the emission in the two images displayed. Nitrogen oxide produces only ultraviolet emission. Nitrogen dioxide produces visible emission, with dominant bands in the green and yellow. Very weak emission was observed with band pass filters at 521, 559, and $619 \mathrm{~nm}$, but showed a similar spatial intensity distribution as in Fig. 6a and 6b. This indicated that the emission at these wavelengths followed the hydrogen/oxygen reactions and were not caused by possible nitrogen entrainment. The exception was a zone of some weak additional green-yellow emission near the throat.

Oscillations were observed during each of the hot-fire tests. Because of the low frequency of imaging, the major frequency component could not be determined. Further investigation would be required to show conclusively whether this behavior is an artifact of the two-dimensionality or generic to the injector design.

\section{Ultraviolet Spectra}

UV spectra of OH near the sleeve exit plane were recorded at five vertical locations, with nondimensional coordinates at $\mathrm{z} / \mathrm{h}=0,0.43,0.65$, 0.87 , and 1.06. Fig. 7 shows two such spectra, at $\mathrm{z} / \mathrm{h}=0.43$ and 0.88 , respectively. Each figure shows a well resolved $\mathrm{OH}$ spectrum with a complex rotational structure. A prominent bandhead at $306.36 \mathrm{~nm}$ can be seen in each spectrum. The intensity distribution over the individual lines reflects the rotational level populations. Rotational temperatures can therefore, in principle, be extracted. Because the collected emission spans the chamber, and with it a wide range of $\mathrm{OH}$ densities and temperatures, only qualitative conclusions can be drawn concerning the temperature and number densities. The spectrum at $\mathrm{z} / \mathrm{h}=0.43$ has a significantly higher intensity then the spectrum at $\mathrm{z} / \mathrm{h}=0.88$. This indicates a higher $\mathrm{OH}$ density at that location. The spectral envelope at $\mathrm{z} / \mathrm{h}=0.43$ shows a maximum intensity at approximately $319.5 \mathrm{~nm}$. The spectral envelope at $\mathrm{z} / \mathrm{h}=\mathbf{0 . 8 8}$ shows a maximum at about $317 \mathrm{~nm}$. The shift to higher rotational line population at $\mathrm{z} / \mathrm{h}=0.43$ indicates that the temperature is higher than at $\mathrm{z} / \mathrm{h}=0.88$, which corresponds with the Raman data obtained earlier.

A qualitative representation of the $\mathrm{OH}$ number density was obtained by integrating the intensity over all vibration-rotation lines. Fig. 8 shows the result for the spectra measured as a function of location. The values were non-dimensionalized with respect to the maximum intensity. The Raman measured temperature, and $\mathrm{O}_{2}$ - and $\mathrm{H}_{2} \mathrm{O}$ number densities are shown for comparison in dashed lines. These values were also nondimensionalized with respect to the maximum value. A surprising feature was the rapid decay of $\mathrm{OH}$ concentration on the outer part of the core, near the wall of the sleeve insert. A likely cause for the observation was the depletion reaction given in Eq. 1, which produced water and light, and was more likely to take place in the outer core region because of the presence of hydrogen. Raman measurements showing a higher water concentration in the outer region of the core agree with this observation.

The $\mathrm{OH}$ concentration closely followed the temperature contour, with a maximum $\mathrm{OH}$ intensity near the temperature maximum. The core was measured to be oxygen rich, thus the chance for the reaction in Eq. 1 to take place was remote. The $\mathrm{OH}$ concentration therefore, was much higher in the core region.

\section{$\underline{\text { Schlieren Visualization }}$}

A typical instantaneous schlieren image of the combustion chamber is shown in Fig. 9. In the figure, the flow moves from the left to the right. The left side of the image corresponds to the sleeve exit.

The brightest regions in the schlieren image indicated the regions with the strongest density gradients because much of the light through those regions was sufficiently deflected to bypass the dot mask. Because the species composition in most of the core region was approximately constant (mostly water and oxygen), the refractive index gradient was caused by the temperature gradient only. In the outer shell of the core and in the film layer, density gradients could also be attributed to molecular species density differences.

The intensity at the centerline throughout the combustion chamber was low for all the schlieren images. This was anticipated because due to 
symmetry considerations, no density gradient was expected to exist at the centerline. Some brighter spots on the centerline indicated that, at least locally, symmetry was not maintained. A maximum schlieren intensity occurred near $\mathrm{z} / \mathrm{h} \sim 0.3$, which corresponded with the maximum temperature gradient location found in the Raman data between $\mathrm{z} / \mathrm{h}=0.2$ and 0.4 . This intensity maximum stayed at constant distance from the centerline throughout the combustion chamber. The intensity maximum increased moving downstream from the sleeve exit, with an axial maximum at approximately halfway between the sleeve exit plane and the start of the chamber convergence. It was not determined whether the intensity maximum at that location was caused by a maximum axial gradient or by the Gaussian intensity distribution of the laser beam.

At the core of the converging section, the schlieren image was dark, indicating that no noticeable temperature gradients existed and that sufficient mixing had occurred to redistribute thermal energy. Near the walls however, starting near the beginning of the convergening section, small pockets of high and low intensity alternated, indicating turbulent reactions. A large region of high and low intensity pockets in the upstream part of the converging section indicated turbulent mixing between fuel-film and oxygenrich core, destroying the wall protecting fuel film.

The intensity distribution at three cross sections of the image in Fig. 9 were analyzed. The locations of these cross sections are indicated by label A, B, and C. At cross section A, 500 temporally consecutive intensity distributions were obtained, representing $0.5 \mathrm{sec}$ of testing. Seventeen of these intensity distribution are shown in Fig. 10 as a function of time. From this plot it was clear that, although fluctuations occurred, no significant change in location of the intensity maxima occurred over the $17 \mathrm{msec}$ duration of this figure. Furthermore, no significant fluctuations occurred between all 500 intensity distributions. This indicates that near the sleeve, no major flow oscillations occur with frequency components between roughly 2 and $1000 \mathrm{~Hz}$. Fig. 10 also shows a non-symmetric behavior, with stronger gradients in the lower part of the combustion chamber.
Figs. $11 \mathrm{a}, \mathrm{b}$, and $\mathrm{c}$ show intensity profiles obtained by averaging 40 consecutive frames, equivalent to $40 \mathrm{msec}$, at cross section $\mathrm{A}, \mathrm{B}$, and $C$, respectively. The sleeve wall locations are outlined at the bottom of all the figures. A clear asymmetry was seen, the cause of which was not determined. The Raman measured temperature profile is shown with a dotted line in Fig. 11a as a comparison.

The local intensity minimum in the center of Fig. 11a corresponded with the minimum measured Raman temperature. No strong gradient existed at the centerline. Propagating outward from the centerline, the intensity increased and reached a maximum at $\mathrm{z} / \mathrm{h}=0.29$, approximately where Raman temperature measurements showed the strongest gradient. The intensity decreased propagating further outward from the core, and showed a local minimum at $\mathrm{z} / \mathrm{h}=0.64$. This darker area was expected because it corresponded with the temperature maximum measured at $\mathrm{z} / \mathrm{h}=\mathbf{0 . 6}$. Continuing further outward, a small increase in intensity was again observed, with a weak local maximum at $\mathrm{z} / \mathrm{h}=0.84$, where again a noticeable temperature gradient existed. This local maximum was only noticeable in the lower part of the image, corresponding to the top part of the thruster. Near the combustion chamber wall, the intensity of the schlieren image near the hydrogen film was at a minimum, as expected from this cold $(290 \mathrm{~K})$ gas region.

Fig. $11 \mathrm{~b}$ shows stronger intensities in the core, indicating higher temperature gradients. Even though the asymmetry also showed up in this profile, strong secondary maxima were observed both in the top and bottom section of the chamber. These secondary maxima suggested high density gradients which seemed to be too far from the wall to be caused by the shear layer between the hydrogen-film and oxygen-rich core. A possible explanation was that the density gradients were caused by ongoing reactions emanating from the sleeve insert.

Fig. 11c shows a maximum near the centerline, indicating the highest temperature gradient. No temperature gradient was anticipated on the centerline based on symmetry considerations. This unexpected observation could have been caused by a flow instability which was previously observed in the emission images, and which could be responsible for the temperature gradient detected in this averaged intensity profile. The 
centerline intensity at this cross section, however, was low as compared to the intensity at cross section B, and therefore represented only a weak temperature gradient.

The intensity at axial location $\mathrm{C}$ decreased rapidly in transverse direction, possibly due to mixing. A local intensity maximum was observed close to the wall. The likely cause was the difference in density between the hydrogen film and the oxygen rich shear layer. This maximum was relatively narrow, indicating a narrow layer of mixing and possibly combustion. The intensity was low again at the wall, where hydrogen still dominated and where apparently no thermal gradient existed. This showed that the hydrogen wall film still retained its integrity at this location in the chamber.

\section{Summary}

Combustion inside the chamber of a $110 \mathrm{~N}$ $\mathrm{GH}_{2} / \mathrm{GO}_{2}$ thruster was analyzed using an optically accessible two-dimensional laboratory model installed in a high altitude chamber. Techniques used to investigate the flow consisted of spontaneous Raman spectral measurements, visible emission imaging, ultraviolet $\mathrm{OH}$ spectroscopy, and high speed schlieren imaging. Correlating the results from a suite of diagnostics provided a more complete understanding of the flow field.

Results from Raman measurements indicated that the fully reacted oxygen rich core flow that the torch ignitor was designed to provide did not occur. The low temperature center flow and high temperature outer shell suggested that reactions occurred in the outer regions of the core flow and that no hydrogen was entrained in the wake behind the bluff spark plug. The fast depletion of the $\mathrm{OH}$ radical in the outer region of the core flow, as measured with UV spectral measurements indicated the presence of unreacted hydrogen. These observations also called into question all the predictions made using a fully mixed and reacted core flow with uniform species, temperature, and velocity distributions.

Emission measurements and schlieren imaging showed that the fuel-film layer retained its integrity throughout the straight section of the combustion chamber. In the upstream part of the converging section, the layer separated from the wall, mixed with the core flow, and formed a turbulent mixing region. This behavior could improve performance but the hot region could reduce thruster life. Further investigation would be required to show conclusively whether this behavior is an artifact of the two-dimensionality or generic to the injector design.

\section{References}

${ }^{1}$ Arrington, L.A., Reed, B.D., and Rivera, Jr., A., "A Performance Comparison of Two Small Rocket Nozzles," NASA TM-107285, AIAA Paper 96-2582, Lake Buena Vista, FL, July 1996).

${ }^{2}$ Richter, G.P. and Price, H.G., "Proven, LongLife Hydrogen/Oxygen Thrust Chambers for Space Station Propulsion, "JANNAF Propulsion Meeting, New Orleans, Aug. 1986. See also NASA TM88822 .

${ }^{3}$ Weiss, J.M., Analysis of Reacting Flowfields in Low-Thrust Rocket Engines and Plumes, Doctoral Thesis, The Pennsylvania State University, December 1992.

4 Robinson, P.J., "Space Station Auxiliary Thrust Chamber Technology", Final Report, NAS3-24398, NASA CR-185296, July 1990.

${ }^{5}$ Arrington, L.A. and Schneider, S.J., "Low Thrust Rocket Test Facility," NASA TM-103206, AIAA Paper 90-2503, July 1990.

${ }^{6}$ James, T. C. and Klemperer, W., "Line Intensities in the Raman Effect of ' $\Sigma$ Diatomic Molecules," J. Chem. Phys., Vol. 31, No. 1, July 1959, pp. 130-134.

${ }^{7}$ Shirley, J.A., "Investigation of the Feasibility of Temperature Profiling Optical Diagnostics in the SSME Pre-Burner," Final Technical Report NAS8-34774, June 1983.

${ }^{8}$ de Groot, W.A., "Temperature and Species Measurements in a Low Thrust Hydrogen/ Oxygen Rocket Engine," J. of Propulsion and Power, Accepted for Publication.

${ }^{9}$ Gaydon, A.G., The Spectroscopy of Flames, $2^{\text {nd }}$ Ed., Chapman and Hall, 1974.

${ }^{10}$ Jones, R.A., de Groot, W.A., Myrabo, L.N., and Nagamatsu, H.T., "Oxygen Temperature and Concentration Measurements in $\mathrm{H}_{2}-\mathrm{O}_{2}$ Rocket Engines, " AlAA Paper 96-0439, Reno, NV, Jan. 15-18, 1996. 

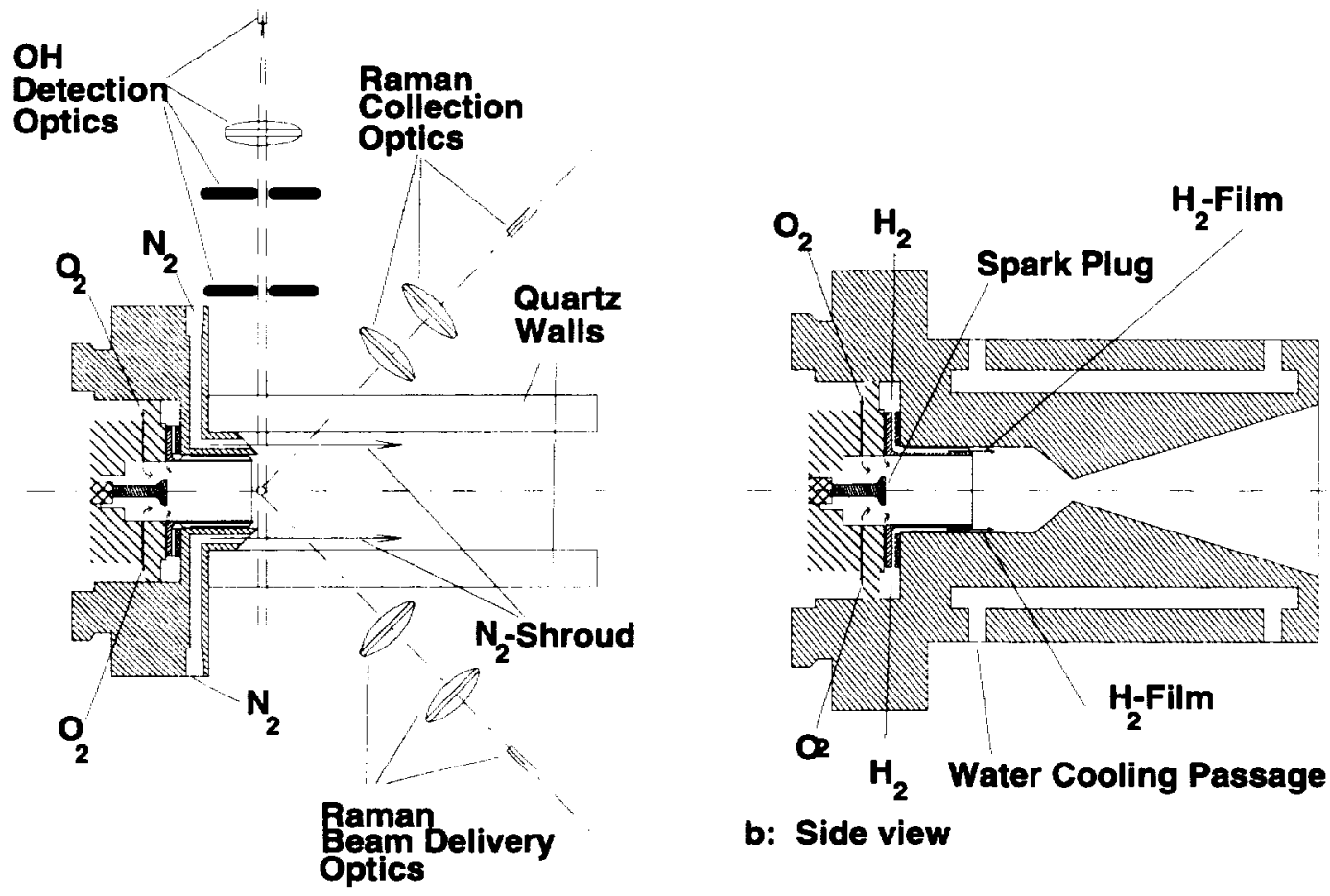

a: Top view and optical arrangement

b: Side view

Figure 1: Schematic of Two-Dimensional Test Hardware and Raman Optics Setup

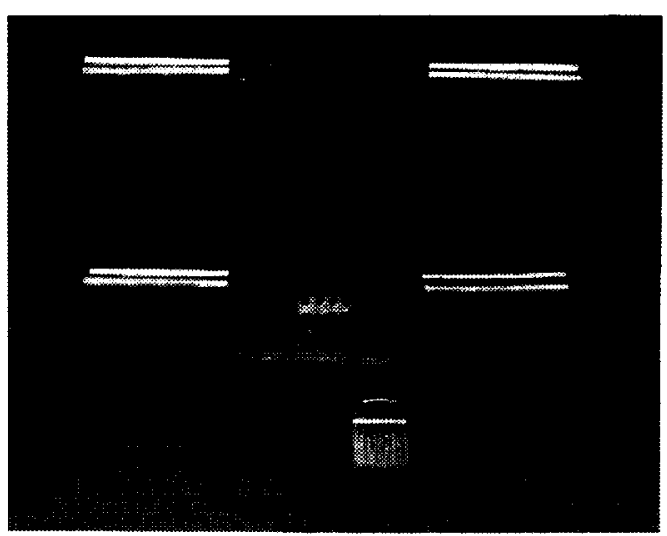

Figure 2: Photograph of Disassembled TwoDimensional Test Hardware Minus Quartz Windows.

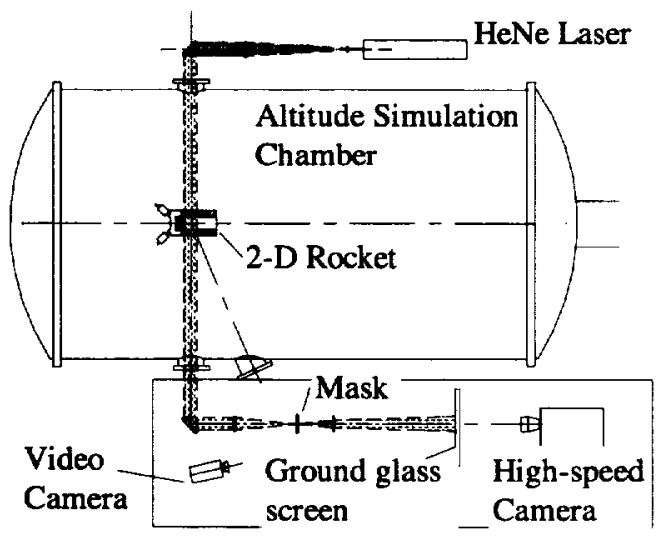

Figure 3: Schematic of Schlieren System 


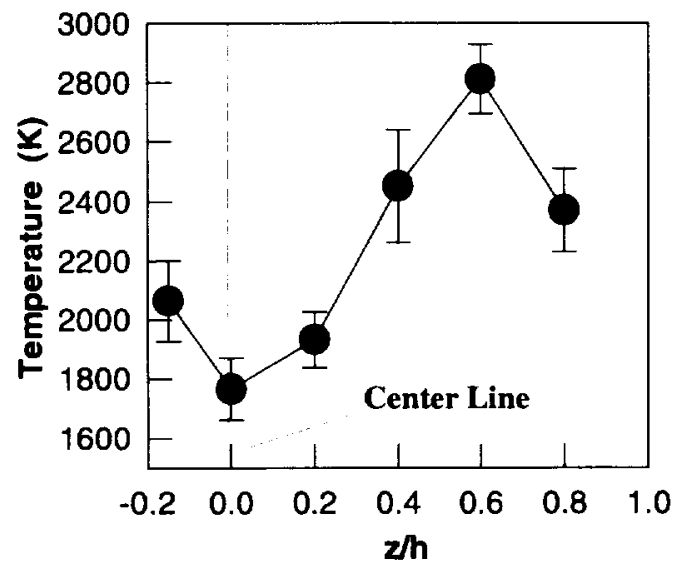

Figure 4: Temperature Profile Measured at Injector Exit
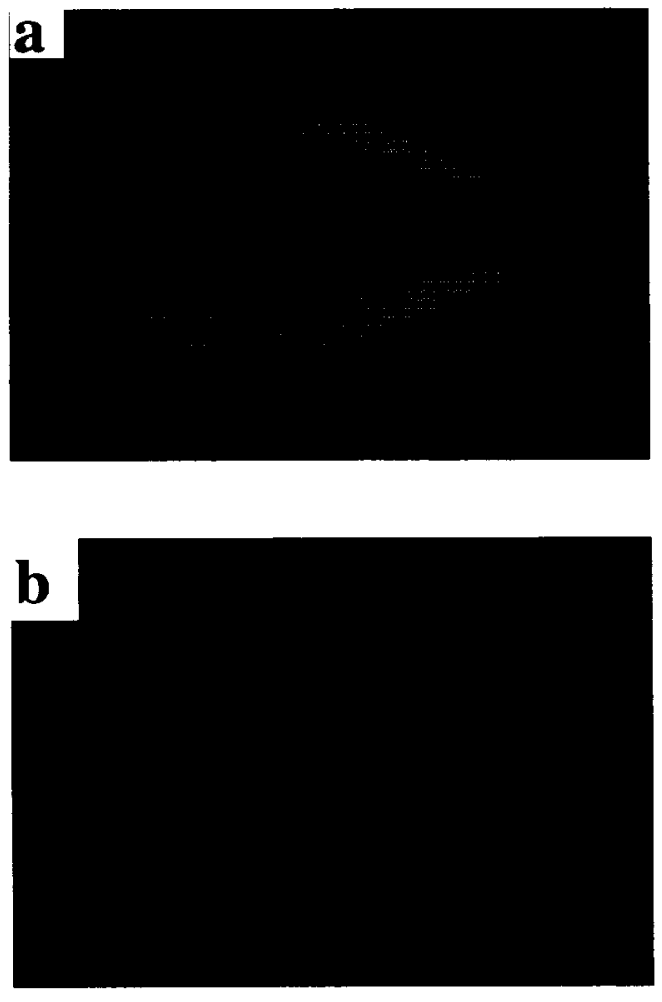

Figure 6: Combustion Chamber Spectral Emission @ a) 479 nm, FWHM = 20 nm b) $630 \mathrm{~nm}$, FWHM $=20 \mathrm{~nm}$

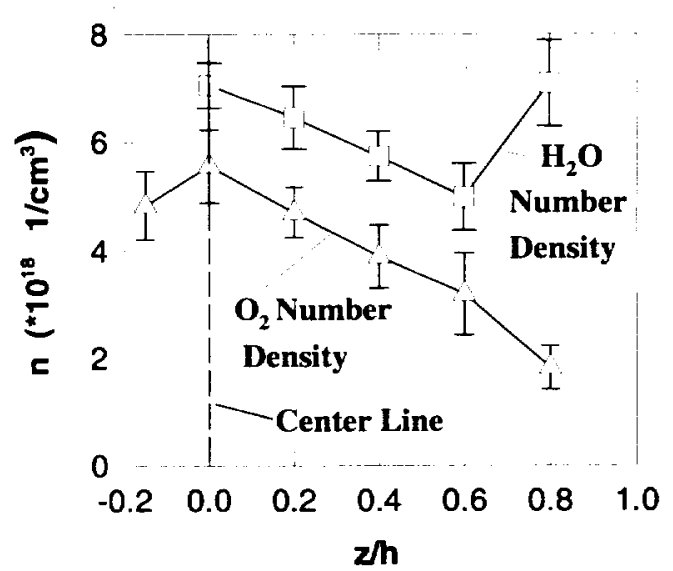

Figure 5: Oxygen and Water Number Density Profiles Measured at Injector Exit.
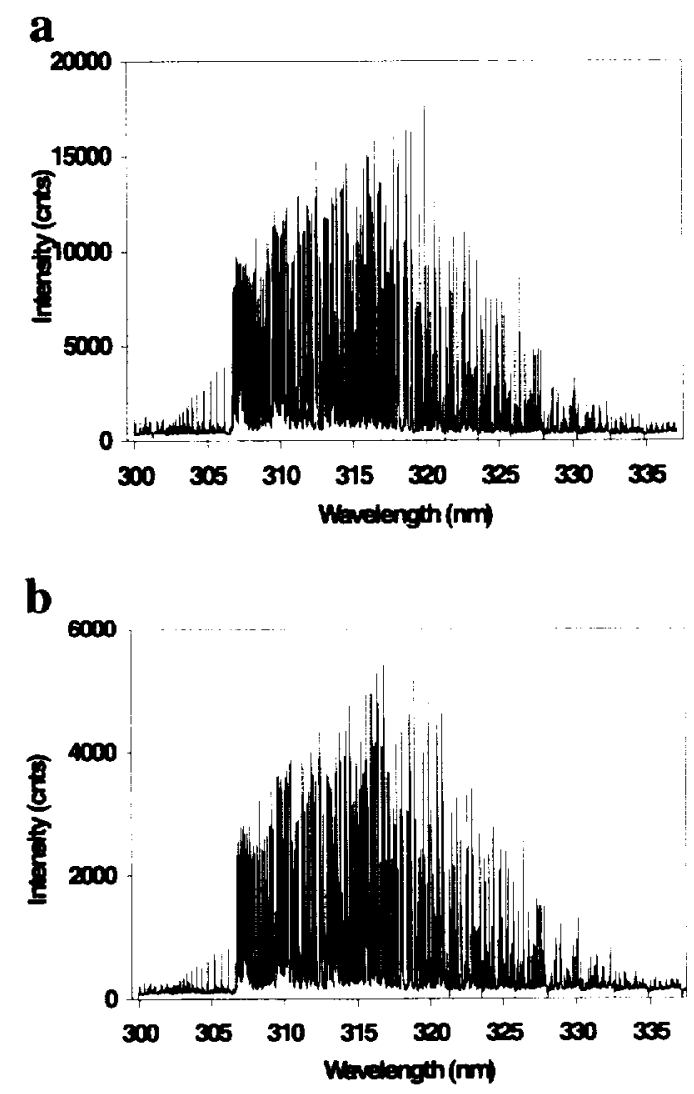

Figure 7: UV Spectrum of $\mathrm{OH}$ at: a) $\mathrm{z} / \mathrm{h}=\mathbf{0 . 4 3}$ b) $\mathrm{z} / \mathrm{h}=\mathbf{0 . 8 8}$ 


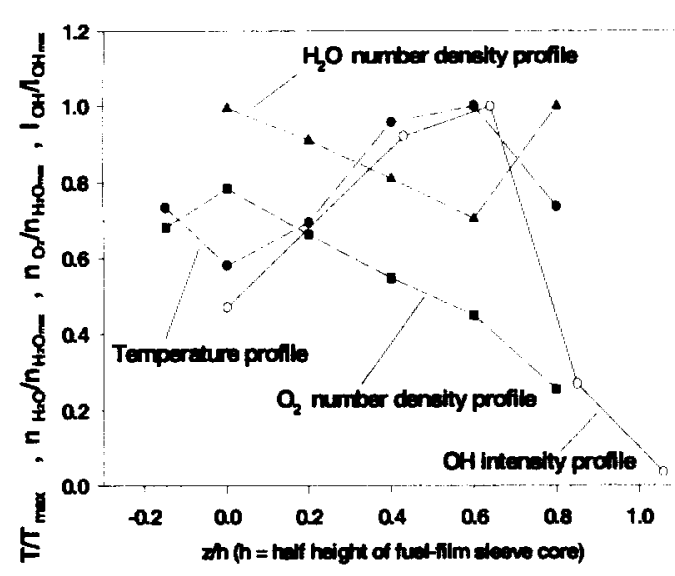

Figure 8:Comparison of Non-Dimensionalized Temperature, Oxygen- and Water- Number Densities from Raman Data, and $\mathrm{OH}$ Emission in the Sleeve Exit Plane.

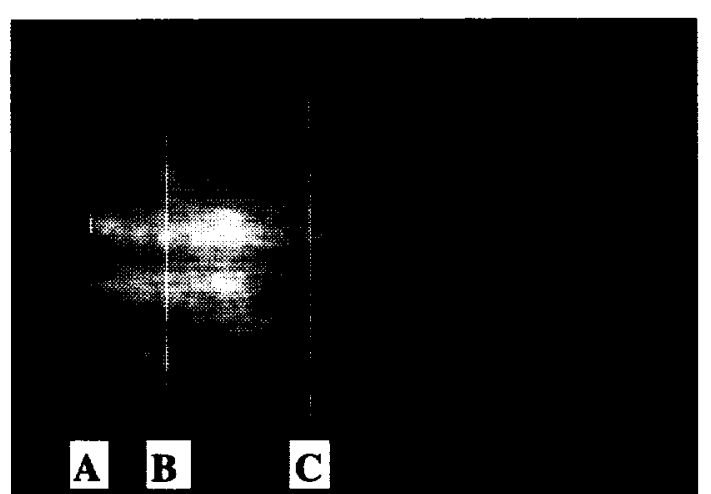

Figure 9: Instantaneous Schlieren Image of Two-Dimensional Chamber.

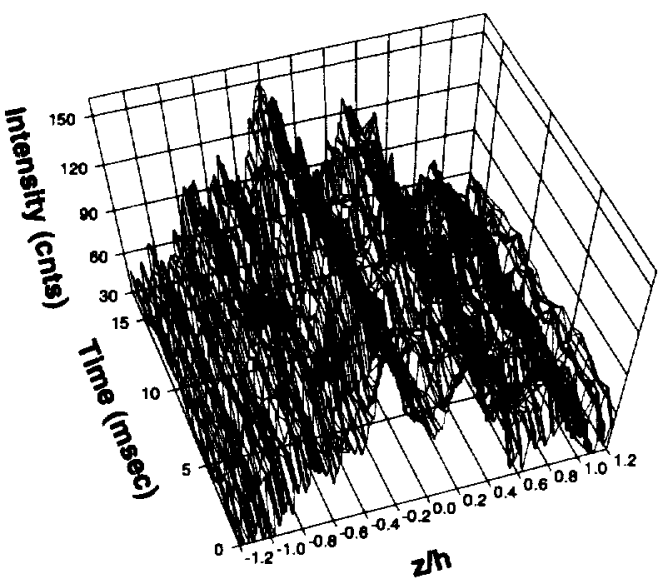

Figure 10: Change of Schlieren Intensity Distribution as a Function of Time at the SleeveExit Plane (Cross Section A).
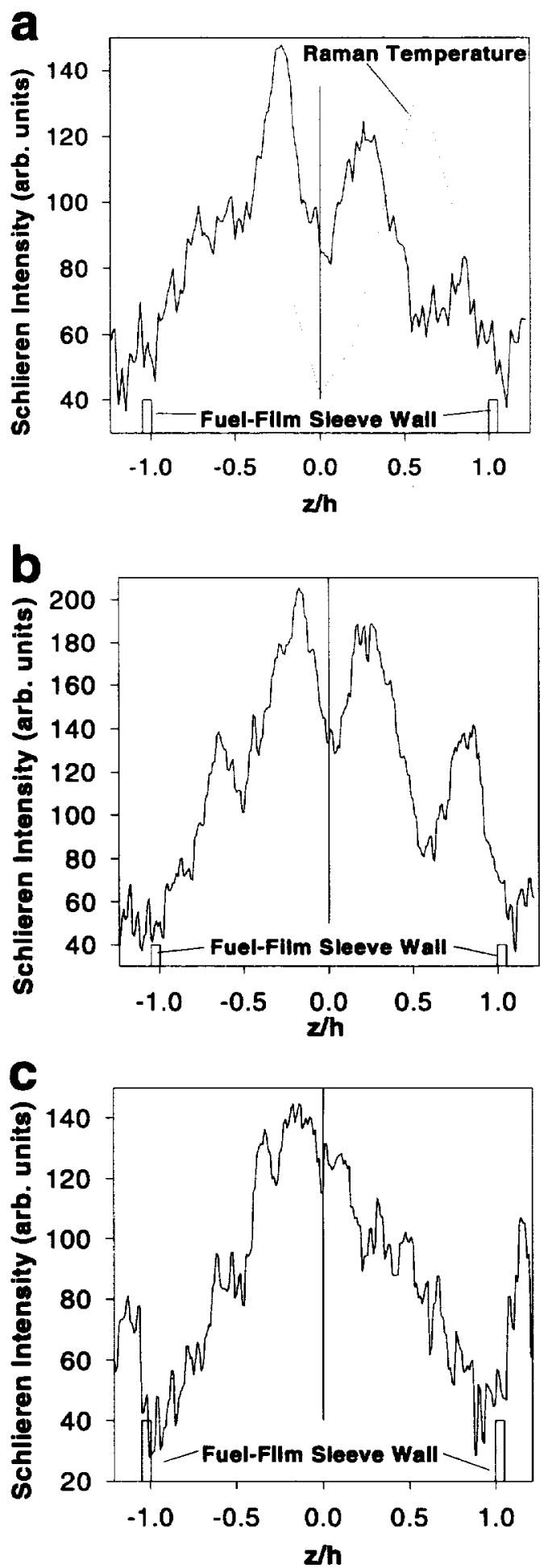

Figure 11: Schlieren Intensity, Averaged Over 40 msec: $\quad$ a) Cross Section $A$

b) Cross Section B

c) Cross Section $\mathrm{C}$ 



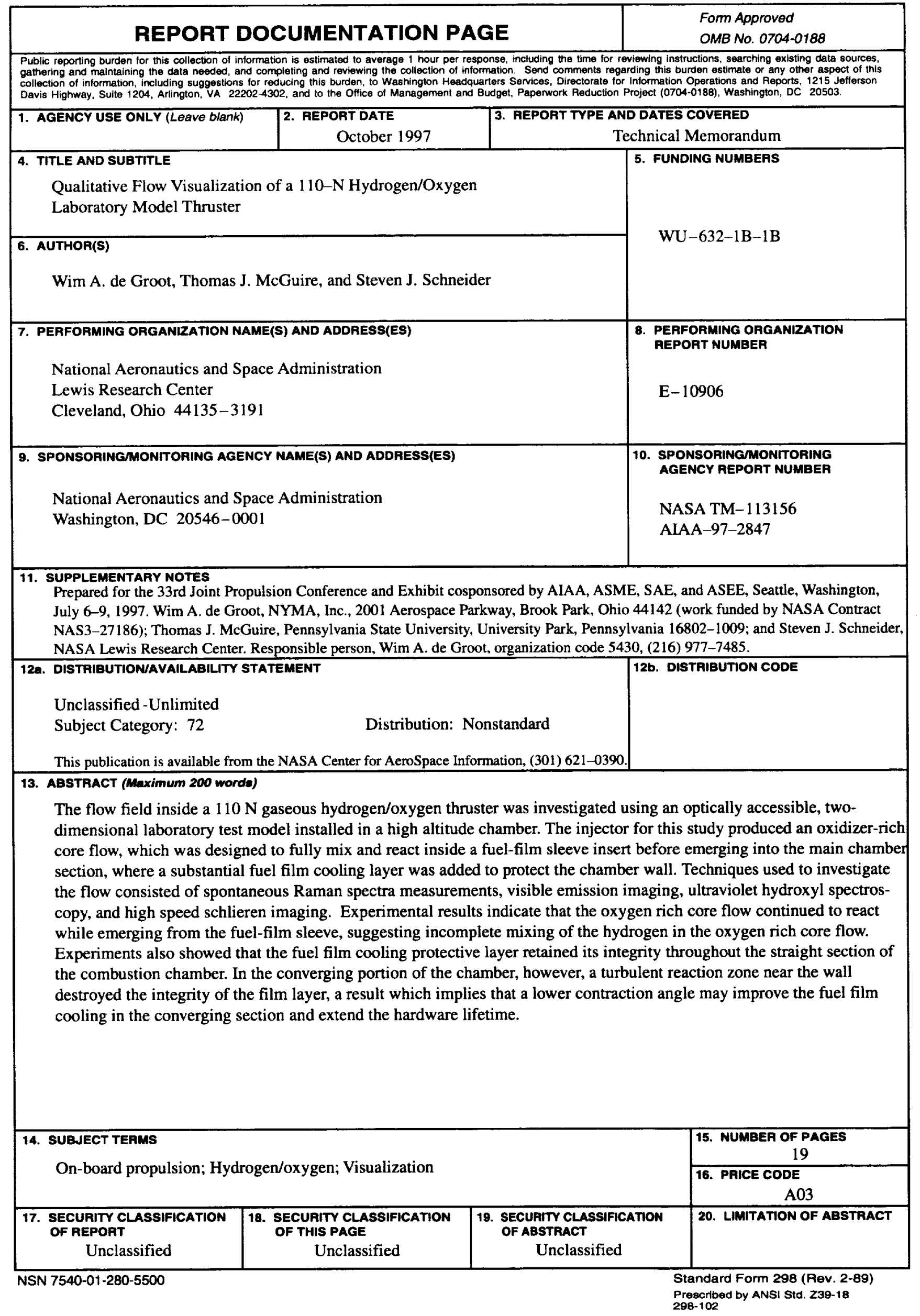

